\title{
Ultrafast Energy Transfer from Solvent to Solute Induced by Subpicosecond Highly Intense THz Pulses
}

\author{
Pankaj Kr. Mishra, ${ }^{\dagger, \ddagger \S}$ Oriol Vendrell, ${ }^{*}, \dagger, \S$ and Robin Santra ${ }^{\dagger, \ddagger}$ \\ ${ }^{\dagger}$ Center for Free-Electron Laser Science, DESY, Notkestr. 85, 22607 Hamburg, Germany \\ ${ }^{\ddagger}$ Department of Physics, University of Hamburg, Jungiusstr. 9, 20355 Hamburg, Germany \\ ${ }^{\S}$ Center for Ultrafast Imaging, University of Hamburg, Luruper Chaussee 149, 22761 Hamburg, Germany
}

Supporting Information

ABSTRACT: The ultrafast energy transfer from an intense, subpicosecond $\mathrm{THz}$ pulse to bulk water at $300 \mathrm{~K}$ and density 1 $\mathrm{g} / \mathrm{cm}^{3}$ is simulated by $\mathrm{ab}$ initio molecular dynamics with explicit inclusion of the laser pulse. A $200 \mathrm{fs}$ subcycle pulse of intensity $5 \times 10^{12} \mathrm{~W} / \mathrm{cm}^{2}$ corresponding to a peak field amplitude of $0.6 \mathrm{~V} / \AA$ and achievable nowadays using optical rectification techniques results in a temperature jump from 300 $\mathrm{K}$ up to $\sim 1000 \mathrm{~K}$ within the first picosecond after the pulse. We discuss in detail the time-dependent structural changes caused by the $\mathrm{THz}$ pulse in the water medium and suggest
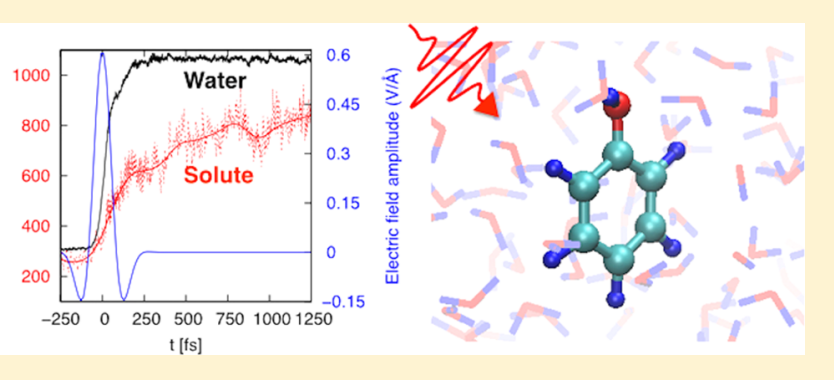
possible ways to measure those changes by pump-probe experimental techniques. The ultrafast energy transfer from the energized water molecules to a solute molecule is studied on a test system, phenol. We find that phenol is, in the gas phase, insensitive to the $\mathrm{THz}$ pulse and only gains energy in solution via collisional energy transfer with the water molecules in its environment. The reason for this is found in the mode of interaction of the THz pulse with the aqueous medium. In short, water molecules respond mainly through their permanent dipole moments trying to orient themselves in the strong electric field of the pulse and disrupting their hydrogen-bonding structure. As compared with the water molecule, phenol has a smaller but still substantial permanent dipole moment. The moments of inertia of phenol are, however, too large for it to rotate in the short duration of the $\mathrm{THz}$ pulse. Therefore, the direct heating-up mechanism is mostly selective to the solvent molecules, whereas the solute heats up indirectly via collisions with its hot environment in about 1 to 2 ps.

\section{INTRODUCTION}

Temperature-jump (T-jump) experiments in liquid water have a long history in the study of chemical kinetics. ${ }^{1-4}$ With the advent of femtosecond lasers a couple of decades ago, ultrafast T-jump experiments became possible and have since been used to study the very fast kinetics of fundamental steps in chemical reactions, ${ }^{5,6}$ folding processes in biomolecules, ${ }^{7-9}$ and fundamental aspects of the hydrogen-bond (H-bond) dynamics and energy transfer in liquid water ${ }^{10-16}$ and other liquids. ${ }^{17} \mathrm{~T}$ jumps in water can be induced, for example, by targeting the first $\mathrm{O}-\mathrm{H}$ vibrational excitation of liquid water at $\sim 3400 \mathrm{~cm}^{-1}$ with an infrared (IR) laser, thus providing T-jumps in the nanosecond to femtosecond time scales. ${ }^{5,6,8,11-15}$ An alternative technique relies on the excitation of dissolved dye molecules with an ultraviolet-visible laser; the dye molecules subsequently release their electronic energy as heat to their environment. ${ }^{9}$ Zewail et al. demonstrated a water temperature increase of up to $20 \mathrm{~K}$ on a picosecond time scale by using an IR pulse of wavelength $1.45 \mu \mathrm{m}$, total pulse energy of $15 \mu \mathrm{J}$, and duration of 5 to $20 \mathrm{ps}^{5}{ }^{\text {Similar }}$ T-jumps have been reported by Hobley et al. using a nanosecond $1.9 \mu \mathrm{m}$ IR pulse ${ }^{14}$ and by Wernet et al. using a femtosecond IR pulse at the wavelength of the $\mathrm{O}-\mathrm{H}$ excitation and duration $60 \mathrm{fs}^{15}$ Nonetheless, $\mathrm{T}$-jumps in water of the order of a few tens of kelvin are still small in comparison with the thermal energy $k_{\mathrm{B}} T$ associated with typical chemical reaction barriers in solution. As an example, a reaction barrier $\Delta E=10 \mathrm{kcal} / \mathrm{mol}$, which should be considered a small barrier in terms of reaction kinetics, corresponds to a temperature of $T=\Delta E / k_{\mathrm{B}} \approx 5000 \mathrm{~K}$. As is well known, this is the fundamental reason why reactive events are a clear example of dynamical rare events.

Here we explore the application of intense, subpicosecond $\mathrm{THz}$ pulses that are able to increase the temperature of bulk water from $300 \mathrm{~K}$ to roughly $1000 \mathrm{~K}$. We investigate the structural properties of the resulting superheated (in the sense that it is not in thermodynamical equilibrium) water phase and how the ultrafast heating-up process of bulk water results in an ultrafast energy redistribution and energy transfer from the superheated solvent to dissolved molecules. We find that the structural properties of water achieved after the short $\mathrm{THz}$ pulse resemble those of supercritical water, that is, water at conditions beyond the critical point at $647 \mathrm{~K}, 22.06 \mathrm{MPa}$, and $0.32 \mathrm{~g} / \mathrm{cm}^{3} ;^{18-20}$ however, here the heating-up process of the medium is isochoric due to the very fast energy transfer from

Received: March 25, 2015

Revised: $\quad$ May 19, 2015

Published: May 22, 2015 
the $\mathrm{THz}$ pulse to bulk water, which results in a transient phase of superheated water still at the usual density of $1 \mathrm{~g} / \mathrm{cm}^{3}$. Supercritical water is known to be an aggressive solvent that can be used, for example, in the degradation of biomaterials and in which organic substances have an increased solubility with respect to water under ambient conditions. ${ }^{21}$

We show that water molecules mainly interact with the $\mathrm{THz}$ pulse through their permanent dipole moments and quickly respond by trying to align along the $\mathrm{THz}$ polarization axis, while $\mathrm{H}$ bonds are broken during this process. The ultrafast energy gain of a solvated molecule in the superheated water environment is determined by collisions with solvent molecules until a quasi-equilibrium is reached. As an example system, we investigate energy transfer to the phenol molecule in solution, which is soluble in water and represents a structural motif common in larger organic and biological systems. Interestingly, whereas the phenol molecule gains a large amount of energy within $1 \mathrm{ps}$ after the $\mathrm{THz}$ pulse due to collisions with hot water molecules, the same pulse has almost no effect on gas-phase phenol. This effect can be traced to the substantially larger moment of inertia of phenol as compared with water and is discussed later in greater detail.

\section{METHODS}

$\mathrm{Ab}$ initio molecular dynamics (AIMD) simulations were performed with the CP2K molecular dynamics package. ${ }^{22,23}$ In this framework, nuclei are described classically and the electronic structure quantum mechanically by means of density functional theory (DFT). ${ }^{24}$ Born-Oppenheimer-based AIMD trajectories were propagated by using the Quickstep electronic structure module ${ }^{23}$ of $\mathrm{CP} 2 \mathrm{~K}$. This module uses the Gaussian and plane waves (GPW) method to solve the self-consistentfield problem. The Perdew-Burke-Ernzerhof $(\mathrm{PBE})^{25}$ functional together with Geodecker-Teter-Hutter $(\mathrm{GTH})^{26}$ pseudopotential was used. The electron density and molecular orbitals were expanded using plane waves with a cutoff of 400 Ry and a TZV2P Gaussian atomic basis set, respectively. Classical trajectories were integrated using a 1 fs time-step, and periodic boundary conditions (PBC) were used in all three spatial directions. Liquid water was described in a cubic box with 256 water molecules at a density of $1 \mathrm{~g} / \mathrm{cm}^{3}$. To obtain a thermalized simulation box, we first optimized the geometrical parameters of the system; then, the temperature was slowly raised up to $300 \mathrm{~K}$ using the Nosé-Hoover thermostat to maintain thermal equilibrium. Subsequently, a production run of 4.5 ps was used to collect 15 initial configurations separated by $300 \mathrm{fs}$, and each initial configuration was then propagated microcanonically (NVE) for $1.5 \mathrm{ps}$ in the presence of the $\mathrm{THz}$ pulse. The inclusion of the electric field under PBC when solving the self-consistent electronic structure equations was performed using the Berry-phase approach. ${ }^{27,28}$ In the case of solvated phenol, a similar simulation protocol was followed, but in this case the phenol molecule was embedded in a periodic box with 256 water molecules.

\section{RESULTS AND DISCUSSION}

Interaction of Liquid Water with the THz Pulse. The electric field of the $\mathrm{THz}$ pulse considered is given by

$$
\mathbf{E}(t)=\epsilon(t) \mathbf{u}_{z} \cos \left(\omega_{\mathrm{c}} t+\phi\right)
$$

where $\epsilon(t)=A \exp \left\{-\left(t-t_{0}\right)^{2} / 2 \sigma^{2}\right\}$ is a Gaussian envelope with $\sigma=84.93$ fs. This corresponds to a full width at half- maximum (fwhm) of $\epsilon^{2}(t)$ of 141 fs. The maximum electric field amplitude $A=0.61 \mathrm{~V} / \AA ̊$ corresponds to a peak power per unit area of $5 \times 10^{12} \mathrm{~W} / \mathrm{cm}^{2}$. We consider a mean frequency $\omega_{\mathrm{c}}$ $=2 \pi \times 3 \mathrm{THz}\left(100 \mathrm{~cm}^{-1}\right)$, which results in a pulse between a half and full cycle long. $\mathbf{u}_{z}$ is the polarization direction of the electric field and $\phi$ is the carrier-envelope phase, which we set equal to $\pi / 2$. In all reported results, the $\mathrm{THz}$ pulse envelope is centered at $t_{0}=0 \mathrm{fs}$ and trajectories are started at $t=-250 \mathrm{fs}$, when the Gaussian envelope of the pulse is still negligible. $\mathrm{THz}$ pulses of these characteristics were generated recently by optical rectification of mid-infrared laser pulses in an organic nonlinear crystal. $^{29}$

Figure 1 shows the average energy per water molecule in its different types of degrees of freedom as a function of time. The

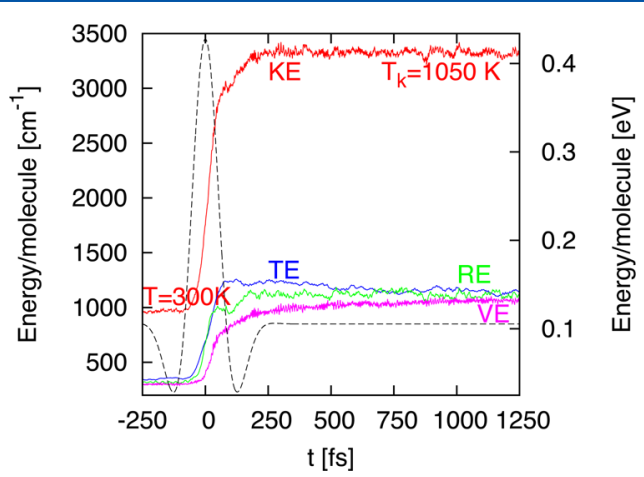

Figure 1. Kinetic energy partitioning per water monomer as a function of time. $t=0$ corresponds to the center of the $\mathrm{THz}$ pulse envelope. Red: total kinetic energy (KE), blue: translational energy (TE), green: rotational energy $(\mathrm{RE})$, pink: vibrational energy (VE), dashed: electric field amplitude. The maximum of the electric field amplitude is 0.61 $\mathrm{V} / \AA$. For water $\langle K E\rangle=(9 / 2) k_{\mathrm{B}} T_{k}$, where $T_{k}$ is just a kinetic temperature and no assumption of thermal equilibration is made whatsoever. $\langle K E\rangle$ is the ensemble-averaged kinetic energy per water molecule from all trajectories and all water molecules.

$\mathrm{THz}$ pulse is centered at $t=0$. The energy transfer from the pulse to water and the subsequent energy redistribution are analyzed by partitioning the total kinetic energy into three contributions: (1) translational energy (TE) of the center of mass of the molecule; (2) rotational energy (RE) around the instantaneous principal axes of rotation; and (3) vibrational energy (VE). VE includes the bending and the two stretching modes of water molecules, which were not separated here. The $\mathrm{TE}$ and RE contributions correspond to hindered rotations and librational motion of water monomers in liquid water. In Figure 1 , the mean value of the total $\mathrm{KE}$ at $t=-250$ fs corresponds to a temperature of $300 \mathrm{~K}$, and the probability distributions of TE, $\mathrm{RE}$, and VE correspond to a Maxwell-Boltzmann distribution also at $300 \mathrm{~K}^{30}$ During the pulse, the $\mathrm{KE}$ per monomer increases most rapidly as the pulse reaches the peak electric field amplitude of 0.5 to $0.6 \mathrm{~V} / \AA$, which is present only during few tens of femtoseconds. The significant increment in $\mathrm{KE}$ starts only after the pulse reaches about half of its maximum amplitude $(\sim 0.25$ to $0.3 \mathrm{~V} / \AA)$ and keeps on increasing until it reduces to less than half of its peak value. As expected, the KE remains constant after the pulse, only slightly fluctuating due to the exchange of total $\mathrm{KE}$ and potential energy. The total energy of each trajectory after the $\mathrm{THz}$ pulse is conserved to numerical accuracy, as expected from a microcanonical simulation. The $\mathrm{KE}$ increase per monomer of $\sim 2300 \mathrm{~cm}^{-1}(0.3 \mathrm{eV}$ or $6.6 \mathrm{kcal} /$ $\mathrm{mol}$ ) corresponds to a final kinetic temperature of $\sim 1050 \mathrm{~K}$. 
This energy gain corresponds to an absorption of $\sim 30 \mathrm{THz}$ photons per water monomer. The increase in VE is slower than TE and RE. The reason is that the THz pulse interacts mainly with the permanent dipole moments of the water monomers, forcing them to rotate out of their preferred arrangement. In the bulk, molecular rotations are hindered and quickly lead to collisions. This sets in motion hindered molecular translations. The resulting collisions quickly bring energy to internal modes on a short time scale. We assume that the heating process is isochoric on a picosecond time scale for a bulk system, meaning on such short time scales no increase in volume is expected to take place. All KE contributions, namely, TE, RE, and VE, reach a quasi-equilibrium distribution within 1 ps after the pulse. In this transient state of the solvent, essentially no H-bond network is present anymore although the system is found at the density of the liquid. ${ }^{30}$

The vibrational structure of liquid water has been characterized by infrared ${ }^{10,31,32}$ and Raman ${ }^{33}$ spectroscopies and by neutron scattering ${ }^{33}$ measurements. Intramolecular features appear in the $1200-4000 \mathrm{~cm}^{-1}$ spectral range. Water molecules in the liquid phase librate with frequencies in the range $400-1200 \mathrm{~cm}^{-1}$ with a maximum at $\sim 670 \mathrm{~cm}^{-1}$. The intramolecular $\mathrm{HOH}$ bending band appears in the range 1200$2200 \mathrm{~cm}^{-1}$ with a clearly distinguishable peak at $\sim 1650 \mathrm{~cm}^{-1}$, and the $\mathrm{OH}$ stretching band appears in the range 2900-3700 $\mathrm{cm}^{-1}$ centered at $\sim 3450 \mathrm{~cm}^{-1}$. The power spectrum or spectral density describes the density of modes per unit frequency and can be obtained as the Fourier cosine transform of the velocity-velocity autocorrelation function $(\mathrm{VACF})^{34}$

$$
\begin{aligned}
\mathbf{S}(\omega, \tau) & =\int_{0}^{\infty} \frac{1}{N} \sum_{j}\left\langle\mathbf{v}_{j}(t+\tau) \cdot \mathbf{v}_{j}(\tau)\right\rangle \exp (-t / \alpha) \cos (\omega t) \mathrm{d} t \\
& =\int_{0}^{\infty} a_{\tau}(t) \exp (-t / \alpha) \cos (\omega t) \mathrm{d} t
\end{aligned}
$$

where $S(\omega, \tau)$ is the spectral density of vibrational modes at frequency $\omega$ and delay time $\tau$ with respect to the THz pulse, whereas $\mathbf{v}_{j}$ is the velocity of the $j$ th atom. Brackets represent ensemble averaging over different initial conditions (realizations). Deviations from $a_{\tau}(t)=0$ for $t \gtrsim 500 \mathrm{fs}$ are assumed to be the result of insufficient sampling over the number of averaged trajectories. Therefore, the $\exp (-t / \alpha)$ function brings the tail of $a_{\tau}(t)$ smoothly to 0 within $\sim 500 \mathrm{fs},{ }^{35}$ which in the frequency domain corresponds to a convolution with a Lorentzian profile. The spectral density is calculated here at different time delays $\tau$ with respect to the center of the $\mathrm{THz}$ pulse.

The spectral density of liquid water as a function of the time delay with respect to the center of the $\mathrm{THz}$ pulse is shown in Figure 2a. In it one can clearly follow in time the strong perturbation of the vibrational structure of liquid water caused by the interaction with the THz pulse. The spectra at $\tau=-750$ and $-250 \mathrm{fs}$ are indistinguishable from the power spectrum of liquid water at room temperature. Right after the pulse, the $\mathrm{OH}$ stretching band at $3430 \mathrm{~cm}^{-1}$ blue shifts to $3620 \mathrm{~cm}^{-1}$ due to disruption of $\mathrm{H}$ bonds, whereas the $\mathrm{HOH}$ bending mode (1648 $\mathrm{cm}^{-1}$ ) does not change significantly. The librational motion band at $\sim 585 \mathrm{~cm}^{-1}$ red shifts to $\sim 333 \mathrm{~cm}^{-1}$ after the pulse. Librational modes are known to be very sensitive to changes in the H-bonding network, ${ }^{36}$ and such red shifts have been observed for water above $T=500 \mathrm{~K}^{37}$ Our results indicate the $\mathrm{T}$-jump produced by the $\mathrm{THz}$ pulse can be followed in time by
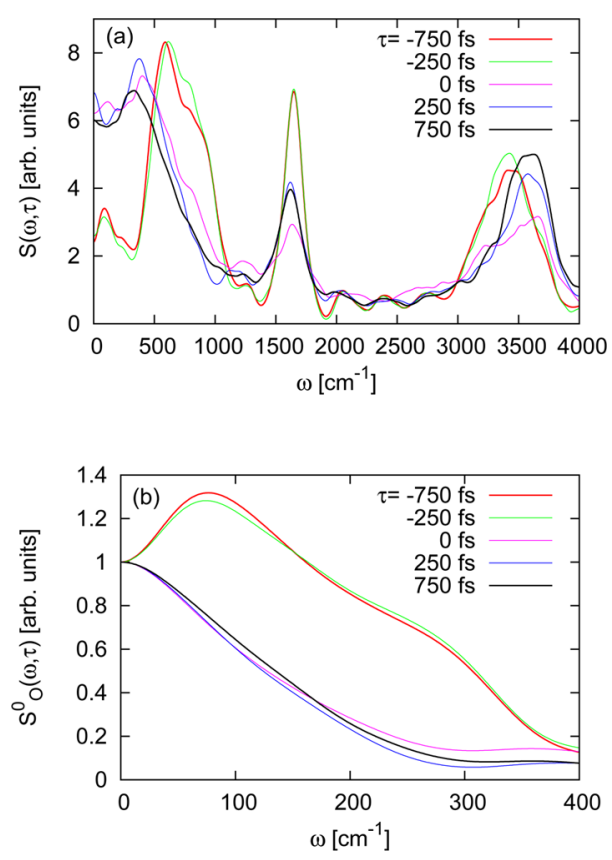

Figure 2. (a) Spectral density of water calculated as the Fourier transform of the velocity-velocity autocorrelation function of all atoms and (b) spectral density calculated from the oxygen atom velocities only. The different time delays $\tau$ correspond the difference between the center of the $\mathrm{THz}$ pulse envelope and the 0 -time for the evaluation of the autocorrelation function. $\tau$ can be interpreted as the delay between the $\mathrm{THz}$ pump pulse and an IR probe pulse.

measuring a transient IR absorption spectrum as a function of the time delay, for example, following the blue shift of the $\mathrm{OH}$ band as the temperature increases within the first picosecond after the $\mathrm{THz}$ pump pulse or alternatively by following the evolution of other spectral features previously described.

In the low-frequency region between 10 and $400 \mathrm{~cm}^{-1}$, Raman, ${ }^{38,39}$ far-infrared, ${ }^{40-42}$ and neutron scattering ${ }^{43,44}$ spectra of liquid water show two weak bands with peaks at $\sim 60$ and $200 \mathrm{~cm}^{-1}$, which correspond to hindered translational modes involving several water molecules. ${ }^{36}$ There has been some debate over the interpretation of the band at $\sim 60$ $\mathrm{cm}^{-1} \cdot 36,38,45$ The widely accepted interpretation is that the existence of the peak at this frequency corresponds to vibrations of the cage structure formed by various water molecules. The band near $\sim 250 \mathrm{~cm}^{-1}$ corresponds to the $\mathrm{O}-\mathrm{O}$ intermolecular stretching vibrations, that is, hindered translations of H-bonded molecules. ${ }^{36}$ The power spectrum $S_{0}^{0}(\omega$, $\tau)$ calculated at different time delays and shown in Figure $2 b$ reveals how the long-range structure of water is changed by the $\mathrm{THz}$ pulse in this low-frequency spectral region. $S^{0}{ }_{\mathrm{O}}(\omega, \tau)$ is obtained from the VACF of the oxygen atoms only and is normalized to 1 at $t=0$. This results in cleaner structural information on the concerted network vibrations of water than by inspecting the $S(\omega, \tau)$ spectral density. ${ }^{36} S^{0}{ }_{\mathrm{O}}(\omega, \tau)$ at -750 and -250 fs time delays shows the two peaks and is consistent with liquid water results at room temperature. ${ }^{38,43}$ Because of the $\mathrm{THz}$ pulse, the mobility of the molecules increases and dramatically changes the network structure. At delay times 0 , 250 , and $750 \mathrm{fs}$, the intensity of the band at centered at 250 $\mathrm{cm}^{-1}$ decreases due to the depletion of the H-bond network. The disappearance of the peak at $60 \mathrm{~cm}^{-1}$ suggests the disruption of any type of cage structure at the level of several water monomers. The power spectrum obtained after the pulse 
is indicative of a very quick loss of correlation between the relative motions of oxygen atoms in the superheated water on the time scale of a few tens of femtoseconds. From the above analysis, one can conclude that the $\mathrm{THz}$ pulse dramatically alters the network structure of liquid water on a subpicosecond time scale. We emphasize that the heating-up process is ultrafast and that the superheated water, right after the pulse, is still found at the density of liquid water under standard conditions.

Energy Transfer to a Solute Molecule. We just discussed how a short and highly intense $\mathrm{THz}$ pulse applied to liquid water results in a large and sudden kinetic energy increase of the water molecules and, consequently, of the temperature of the medium, mostly by coupling to the permanent dipole moments of the individual monomers and disrupting the $\mathrm{H}$ bond structure of the liquid phase. In the following, we investigate in some detail the mechanism by which a solute molecule gains energy in this environment. As a test solute system, we consider one phenol molecule placed in a simulation box with 256 water molecules. In the following, we compare the $\mathrm{THz}$ response of a phenol molecule in vacuum and in solution to characterize the differential effect that the aqueous environment has on the ultrafast heating process.

The total kinetic energy of phenol is partitioned into three contributions, similarly as before for water, to analyze the energy redistribution to various types of degrees of freedom. Becausre phenol is a neutral molecule, its center of mass does not interact with a spatially homogeneous electric field, and in vacuum the three translational degrees of freedom (DOF) conserve their energy. Therefore, these are not shown. Figure $3 a$ presents the effect of the pulse on the energy of the hindered translational and rotational degrees of freedom as compared with the rotational energy of phenol in vacuum. Interestingly, the increase in rotational energy (RE) in vacuum is negligible in comparison with the increase in $\mathrm{RE}$ and in translational energy (TE) of the center of mass in solution. The permanent dipole moment of an isolated water monomer is $1.85 \mathrm{D},{ }^{46}$ whereas for isolated phenol, it is $1.224 \mathrm{D} .^{47}$ So, it may be at first instance striking that rotations of the phenol molecule are insensitive to the $\mathrm{THz}$ pulse in the gas phase as compared with an isolated water molecule, ${ }^{30}$ at least in view of their not so different permanent dipole moments. For water, the interaction of the monomer's permanent dipole moment with the pulse accounts for a large part of the interaction with the electromagnetic field. The main difference lies in their different rotational constants (or moments of inertia). The three rotational constants are $(27.87,14.51,9.28) \mathrm{cm}^{-1}$ for a water molecule ${ }^{48}$ and $(0.19$, $0.09,0.06) \mathrm{cm}^{-1}$ for a phenol molecule. ${ }^{47}$ The phenol molecule does not respond to the $\mathrm{THz}$ pulse because it is too massive to start rotating within the short pulse duration. In general, many soluble molecules in water will not be able to directly be affected by such a $\mathrm{THz}$ pulse, either because of having a small permanent dipole moment, larger moments of inertia than water, or both. This makes the energy-transfer mechanism to an aqueous medium with solute molecules using a short and intense subcycle $\mathrm{THz}$ pulse selective to the molecules of the solvent, which first heat up and then transfer energy to the solute molecules via frequent collisions. During and after the $\mathrm{THz}$ pulse, the RE and TE energy contributions of the solvated phenol molecule steadily increase. These motions basically correspond to displacements of the solvated molecule relative to the solvation shell. After the $\mathrm{THz}$ pulse, each rotational and translational degree of freedom has increased its mean energy
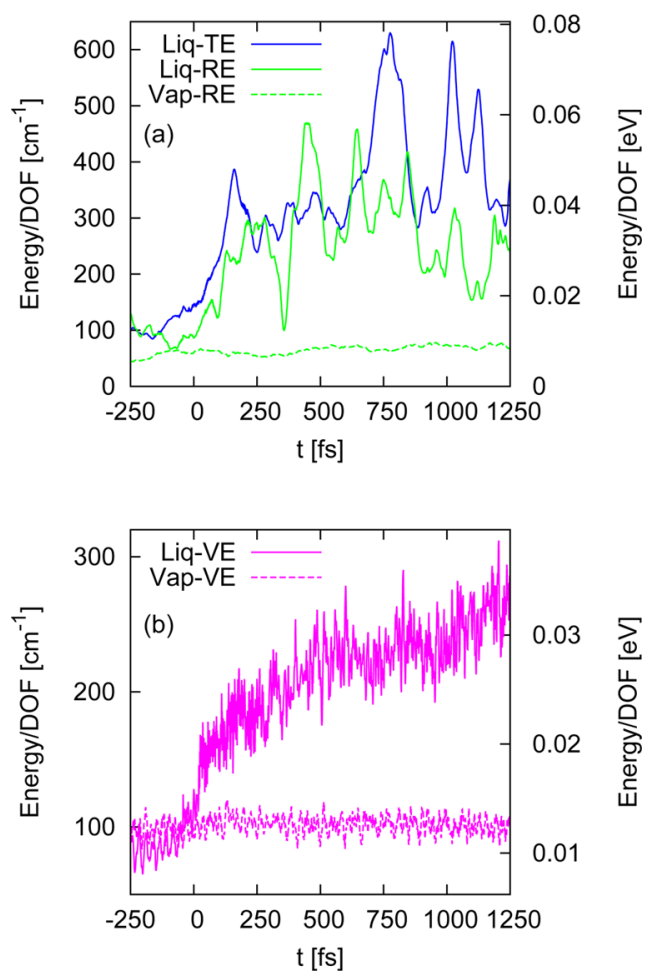

Figure 3. Average kinetic energy of the phenol molecule in different types of degrees of freedom. (a) Translational energy (TE) of the center of mass in the liquid (blue, solid), rotational energy (RE) in the liquid (green, solid) and in vacuum (green, dashed). (b) Vibrational energy (VE) in solution (pink, solid) and in vacuum (pink, dashed).

by 150 to $200 \mathrm{~cm}^{-1}$ within 1 ps after the pulse. The strong energy variations seen in Figure $3 \mathrm{a}, \mathrm{b}$ result from the limited number of sampled trajectories. A better sampling was not possible due to their high computational cost.

Similarly, the internal vibrational modes of isolated phenol are insensitive to the intense $\mathrm{THz}$ pulse, which is seen in the negligible energy increase of the vibrational energy of phenol in vacuum shown in Figure 3b. Therefore, the vibrational energy increase in the solute is mostly a consequence of collisions with energized solvent molecules. In total, phenol increases its total kinetic energy by more than $\sim 8400 \mathrm{~cm}^{-1}$ in the liquid environment as compared with the interaction with the pulse without mediation by the solvent molecules. The heating-up mechanism is clearly illustrated in Figure 4a, where the average energy increase per DOF is compared for isolated water, isolated phenol, as well as bulk water and solvated phenol in water environment. Trajectories of an isolated water molecule, an isolated phenol molecule, and a solvated phenol molecule in the presence of the $\mathrm{THz}$ pulse are provided as Supporting Information. In the isolated water case, we see that the kinetic energy of the molecule increases within the pulse by a similar amount as for water molecules in the bulk. Before the pulse is over, the rotational motion of the water molecule is slowed down again by the pulse by some amount, which is a particularity of the initial conditions of the trajectory shown. Isolated phenol in the gas phase is insensitive to the pulse as previously discussed, while solvated phenol quickly gains energy from its aqueous environment. Figure $4 \mathrm{~b}$ shows the kinetic temperature increase in the solvent water molecules and of the phenol molecule. The temperature of the phenol molecule quickly increases shortly after the surrounding solvent 

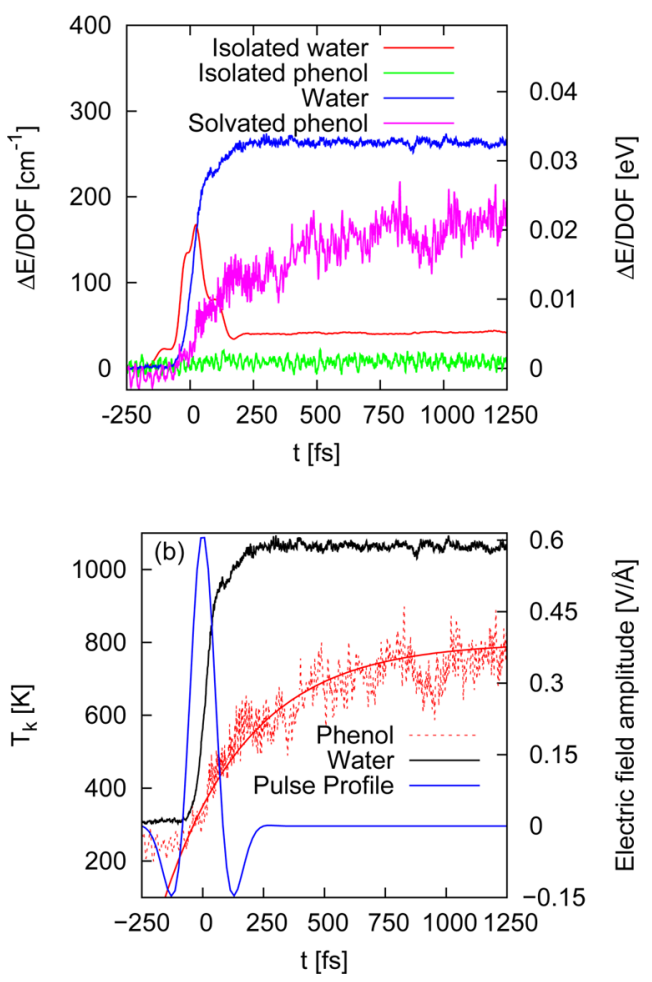

Figure 4. (a) Average kinetic energy increment $(\Delta E)$ in each degree of freedom of isolated water (red), isolated phenol (green), bulk water (blue), and solvated phenol in water (magenta). (b) Kinetic temperature $\left(T_{\mathrm{K}}\right)$ of water and the dissolved phenol molecule. Water molecules gain energy only for the duration of the $\mathrm{THz}$ pulse. The energy of the solvated phenol molecule keeps increasing after the $\mathrm{THz}$ pulse due to collisions with water molecules. The solid curve along the phenol dotted red line corresponds to a fit of the form $T(t)$ $=T_{0}+\Delta T_{\mathrm{f}}\left(1-\exp \left(\ln (2) t / t_{1 / 2}\right)\right)$ for the heating-up kinetics.

molecules start to gain energy, as seen in Figure $4 \mathrm{~b}$. About half of the temperature increment during the first picosecond takes place after the $\mathrm{THz}$ pulse is over, and the final kinetic temperature of phenol reaches about $750 \mathrm{~K}$ within 1 ps after the $\mathrm{THz}$ pulse. A heating-up curve of the form $T(t)=T_{0}+$ $\Delta T_{\mathrm{f}}\left(1-\exp \left(\ln (2) t / t_{1 / 2}\right)\right)$ with $t_{1 / 2}=242 \mathrm{fs}, T_{0}=350 \mathrm{~K}$, and $\Delta T_{\mathrm{f}}=450 \mathrm{~K}$ has been fit to the heating-up curve of phenol obtained from the AIMD simulations. This indicates a fast initial energy transfer and corresponding associated temperature increase between 400 and $500 \mathrm{~K}$; however, the asymptotic temperature reached within the simple first-order kinetics model is still $>200 \mathrm{~K}$ away from the final temperature of the bulk. From the length of the propagated trajectories, one cannot infer the time scale of the final equilibration to the temperature of the surrounding water molecules.

Solvation Dynamics during and after the THz Pulse. As a consequence of the $\mathrm{THz}$ pump pulse, the mobility of the water molecules around phenol increases. The more energetic water molecules can overcome to a larger extent the repulsive short-range potential with the phenol molecule and consequently the average distance between phenol and neighboring water molecules decreases. The nearest distance between carbon atoms of phenol and oxygen atoms of water $\left(d_{\mathrm{C}-\mathrm{O}}\right)$ is shown in Figure $5 \mathrm{a}$ as a function of time. During the pulse, $d_{\mathrm{C}-\mathrm{O}}$ changes from a time-averaged value of $3.38 \AA$ to a minimum of $3.12 \AA$ and then keeps oscillating around a value of $\sim 3.2$ A. By comparing the $d_{\mathrm{C}-\mathrm{O}}$ graphic and the energy increase in water shown in Figure $4 \mathrm{~b}$, one sees that the shortening of
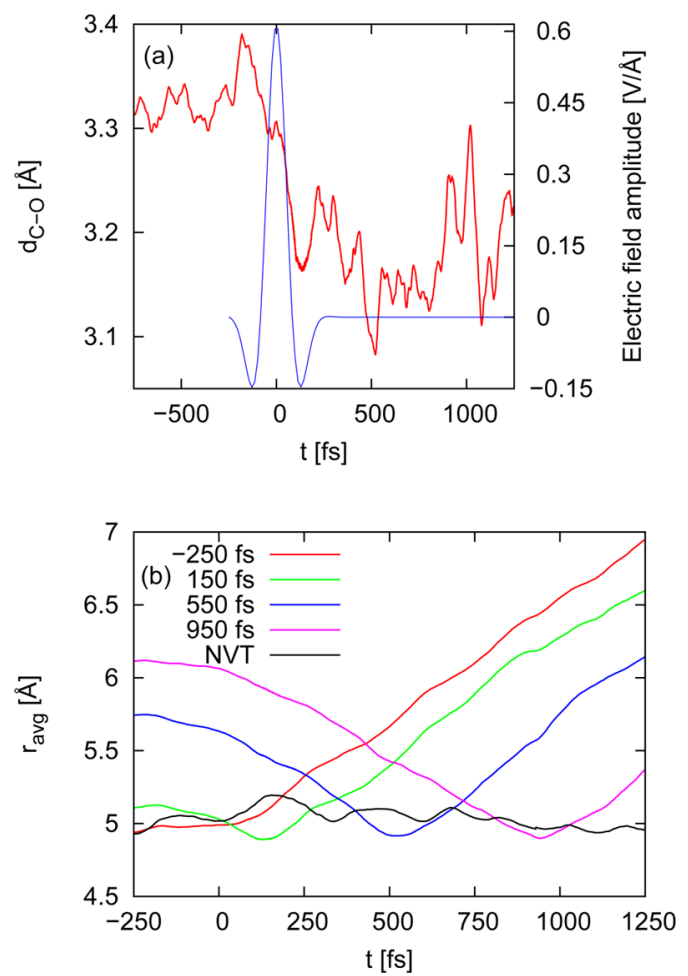

Figure 5. (a) Average value of the nearest distance between carbon and oxygen atoms (red) and electric field amplitude of the $\mathrm{THz}$ pulse (blue). Only oxygen atoms of the solvent are considered. (b) Average distance between a set of water molecules and the center of phenol ring with time. At each given time the reference set of water molecules was found within the spherical region of $6.0 \AA$ around the center of the phenol ring.

$d_{\mathrm{C}-\mathrm{O}}$ is insensitive to the particular temporal profile of the $\mathrm{THz}$ pulse applied and seems to be a function only of the amount of energy per water molecule. This indicates again that the energytransfer mechanism works by directly transferring energy to bulk water molecules and not by triggering directly the motion of solute-solvent vibrational modes, that is, displacements of the phenol molecule in its solvation box.

Water molecules in the first solvation shell of phenol exchange at a large rate under the conditions reached after the pulse, whereas there is practically no exchange of the molecules in the first solvation shell at $300 \mathrm{~K}$ during the time scale of a few picoseconds. Figure $5 \mathrm{~b}$ shows, as a function of time, the average distance between the center of the phenol ring and the centers of the set of water molecules found within a radius of 6 $\AA$ of the center of phenol ring at a particular time delay and compares this distance with its equilibrium value. The rapid exchange of neighbors means that phenol is at all times in contact with very energetic water molecules coming and going from the bulk instead of being surrounded by a static solvation shell. This observation may have consequences, for example, in the activation of chemical processes in which one or more water molecules may have to play an active role in a chemical reaction. A structured water environment at room temperature activates chemical processes by fluctuations that affect the relative potential energy of reactants, transition state, and products geometry. ${ }^{49}$ Instead, the superheated environment resulting from the interaction with the $\mathrm{THz}$ pulse behaves rather like an idealized environment of hard spheres, which can only activate chemical processes by direct collisions and the 
transfer of kinetic energy, as is known from chemical reactions occurring within unstructured environments of rare-gas atoms. $^{50}$

\section{CONCLUSIONS AND OUTLOOK}

A highly intense $\mathrm{THz}$ pulse delivering a power per unit area of $5 \times 10^{12} \mathrm{~W} / \mathrm{cm}^{2}$ to room-temperature bulk water on a subpicosecond time scale results in a large energy increase in the water molecules, to a large extent by interaction with their individual permanent dipole moments, which results in a fast breaking-up of the $\mathrm{H}$-bond structure in the bulk. $\mathrm{THz}$ pulses of these characteristics have been recently realized using optical rectification of mid-infrared laser pulses in a nonlinear organic crystal. ${ }^{29}$ According to our ab initio molecular dynamics simulations, each water monomer increases its energy during the pulse by $\sim 2300 \mathrm{~cm}^{-1}$, which gives rise to a kinetic temperature increase from 300 to $\sim 1050 \mathrm{~K}$. Most of the energy increase occurs within a time window of $\sim 100 \mathrm{fs}$, during which the pulse reaches its peak amplitude of $0.6 \mathrm{~V} / \AA$. Hindered translational and rotational vibrational modes of the water monomers gain energy rapidly during the duration of the $\mathrm{THz}$ pulse, and internal vibrations $(\mathrm{HOH}$ bending and $\mathrm{OH}$ stretching) follow with a delay of a few tens of femtoseconds through collisions between monomers. The power spectrum of the water vibrations calculated at different time delays with respect to the center of the $\mathrm{THz}$ pulse indicates a blue shift of the $\mathrm{OH}$ stretching band from $\sim 3400$ to $\sim 3600 \mathrm{~cm}^{-1}$. This effect is due to the break up of $\mathrm{H}$ bonds in the superheated water phase and might be followed by probing the absorption of the medium in this spectral range by a $\mathrm{THz}$ pump-IR probe scheme. The disappearance of the structure in the power spectrum in the low-frequency region $\left(0-400 \mathrm{~cm}^{-1}\right)$ indicates the depletion of the $\mathrm{H}$-bond network and of any transient structures involving several water molecules, which are present at lower temperatures.

The superheated water phase reached after the $\mathrm{THz}$ pulse quickly transfers energy to a solute molecule, in this example, the phenol molecule, due to constant collisions. As already discussed, water molecules respond to the electric field of the pulse mostly through their individual permanent dipole moments, and a small moment of inertia is also needed to quickly respond and rotate. In contrast, a phenol molecule remains insensitive to the $\mathrm{THz}$ pulse when in vacuum due to its higher moment of inertia with respect to water, although its permanent dipole moment is on the same order of magnitude and only $<50 \%$ smaller. In water, the solvated phenol molecule experiences an energy increase of, on average, about $200 \mathrm{~cm}^{-1}$ per DOF after the pulse. This corresponds to a T-jump from 300 up to $800 \mathrm{~K}$ within 1 ps after the $\mathrm{THz}$ pulse.

The transient conditions of temperature and pressure reached in water after the pulse are beyond the critical point at $647 \mathrm{~K}$ and $22 \mathrm{MPa}$ but still at the liquid density of $1 \mathrm{~g} / \mathrm{cm}^{3}$. The possibility of producing superheated water by application of strong $\mathrm{THz}$ pulses, which quickly initiate intermolecular collisions at a much increased rate as compared with room temperature, may open new avenues in the study of the ultrafast dynamics of thermally activated processes of chemical and biochemical reactions.

\section{ASSOCIATED CONTENT}

\section{(S) Supporting Information}

Effect of decay function on power spectrum and animated trajectories showing the interaction of water and phenol molecules with the $\mathrm{THz}$ pulse. The Supporting Information is available free of charge on the ACS Publications website at DOI: $10.1021 /$ acs.jpcb.5b02860.

\section{AUTHOR INFORMATION}

\section{Corresponding Author}

*E-mail: oriol.vendrell@cfel.de.

\section{Notes}

The authors declare no competing financial interest.

\section{ACKNOWLEDGMENTS}

We are grateful to the Virtual Institute of the Helmholtz Association "Dynamic Pathways in Multidimensional Landscapes" for financial support and to the Juelich Supercomputing Centre of the Forschungszentrum Juelich for computing time on the Juropa cluster through project NIC 5714.

\section{REFERENCES}

(1) Muller, N. Kinetics of Micelle Dissociation by Temperature-Jump Techniques. Reinterpretation. J. Phys. Chem. 1972, 76, 3017-3020.

(2) Rigler, R.; Rabl, C.; Jovin, T. M. A Temperature Jump Apparatus for Fluorescence Measurements. Rev. Sci. Instrum. 1974, 45, 580-588.

(3) Knoll, W.; Stark, G. Temperature-Jump Experiments on Thin Lipid Membranes in the Presence of Valinomycin. J. Membr. Biol. 1977, 37, 13-28.

(4) Tsong, T. Y.; Kingsley, E. Hemolysis of Human Erythrocyte Induced by a Rapid Temperature Jump. J. Biol. Chem. 1975, 250, 786789.

(5) Ma, H.; Wan, C.; Zewail, A. H. Ultrafast T-Jump in Water: Studies of Conformation and Reaction Dynamics at the Thermal Limit. J. Am. Chem. Soc. 2006, 128, 6338-6340.

(6) Ma, H.; Wan, C.; Zewail, A. H. Dynamics of Ligand Substitution in Labile Cobalt Complexes Resolved by Ultrafast T-jump. Proc. Nat. Acad. Sci. 2008, 105, 12754-12757.

(7) Bunagan, M. R.; Yang, X.; Saven, J. G.; Gai, F. Ultrafast Folding of a Computationally Designed Trp-Cage Mutant: Trp2-Cage. J. Phys. Chem. B 2006, 110, 3759-3763.

(8) Ma, H.; Wan, C.; Wu, A.; Zewail, A. H. DNA Folding and Melting Observed in Real Time Redefine the Energy Landscape. Proc. Natl. Acad. Sci. U.S.A. 2007, 104, 712-716.

(9) Phillips, C. M.; Mizutani, Y.; Hochstrasser, R. M. Ultrafast Thermally Induced Unfolding of RNase A. Proc. Natl. Acad. Sci. U.S.A. 1995, 92, 7292-7296.

(10) Lock, A. J.; Bakker, H. J. Temperature Dependence of Vibrational Relaxation in Liquid H2O. J. Chem. Phys. 2002, 117, $1708-1713$.

(11) Huse, N.; Ashihara, S.; Nibbering, E. T.; Elsaesser, T. Ultrafast Vibrational Relaxation of O-H Bending and Librational Excitations in Liquid H2O. Chem. Phys. Lett. 2005, 404, 389-393.

(12) Wang, Z.; Pang, Y.; Dlott, D. D. Hydrogen-Bond Disruption by Vibrational Excitations in Water. J. Phys. Chem. A 2007, 111, 31963208.

(13) Ashihara, S.; Huse, N.; Espagne, A.; Nibbering, E. T. J.; Elsaesser, T. Ultrafast Structural Dynamics of Water Induced by Dissipation of Vibrational Energy. J. Phys. Chem. A 2007, 111, 743746.

(14) Hobley, J.; Kuge, Y.; Gorelik, S.; Kasuya, M.; Hatanaka, K.; Kajimoto, S.; Fukumura, H. Water Expansion Dynamics After Pulsed IR Laser Heating. Phys. Chem. Chem. Phys. 2008, 10, 5256-5256.

(15) Wernet, P.; Gavrila, G.; Godehusen, K.; Weniger, C.; Nibbering, E. T. J.; Elsaesser, T.; Eberhardt, W. Ultrafast Temperature Jump in Liquid Water Studied by a Novel Infrared Pump-X-ray Probe Technique. Appl. Phys. A: Mater. Sci. Process. 2008, 92, 511-516.

(16) Backus, E. H. G.; Tielrooij, K.-J.; Bonn, M.; Bakker, H. J. Probing Ultrafast Temperature Changes of Aqueous Solutions with Coherent Terahertz Pulses. Opt. Lett. 2014, 39, 1717-1720. 
(17) Tokmakoff, A.; Sauter, B.; Fayer, M. D. Temperature Dependent Vibrational Relaxation in Polyatomic Liquids: Picosecond Infrared Pump-Probe Experiments. J. Chem. Phys. 1994, 100, 9035.

(18) Wernet, P.; Testemale, D.; Hazemann, J.-L.; Argoud, R.; Glatzel, P.; Pettersson, L. G. M.; Nilsson, A.; Bergmann, U. Spectroscopic Characterization of Microscopic Hydrogen-Bonding Disparities in Supercritical Water. J. Chem. Phys. 2005, 123, 154503.

(19) Sit, P. H.-L.; Bellin, C.; Barbiellini, B.; Testemale, D.; Hazemann, J.-L.; Buslaps, T.; Marzari, N.; Shukla, A. Hydrogen Bonding and Coordination in Normal and Supercritical Water from Xray Inelastic Scattering. Phys. Rev. B 2007, 76, 245413.

(20) Sahle, C. J.; Sternemann, C.; Schmidt, C.; Lehtola, S.; Jahn, S.; Simonelli, L.; Huotari, S.; Hakala, M.; Pylkkänen, T.; Nyrow, A.; et al. Microscopic Structure of Water at Elevated Pressures and Temperatures. Proc. Natl. Acad. Sci. U.S.A. 2013, 110, 6301-6306.

(21) Akiya, N.; Savage, P. E. Roles of Water for Chemical Reactions in High-Temperature Water. Chem. Rev. 2002, 102, 2725-2750.

(22) Kühne, T. D.; Krack, M.; Mohamed, F. R.; Parrinello, M. Efficient and Accurate Car-Parrinello-like Approach to BornOppenheimer Molecular Dynamics. Phys. Rev. Lett. 2007, 98, 066401.

(23) VandeVondele, J.; Krack, M.; Mohamed, F.; Parrinello, M.; Chassaing, T.; Hutter, J. Quickstep: Fast and Accurate Density Functional Calculations Using a Mixed Gaussian and Plane Waves Approach. Comput. Phys. Commun. 2005, 167, 103-128.

(24) Kohn, W.; Sham, L. J. Self-Consistent Equations Including Exchange and Correlation Effects. Phys. Rev. 1965, 140, A1133A1138.

(25) Perdew, J. P.; Burke, K.; Ernzerhof, M. Generalized Gradient Approximation Made Simple. Phys. Rev. Lett. 1996, 77, 3865-3868.

(26) Goedecker, S.; Teter, M.; Hutter, J. Separable Dual-Space Gaussian Pseudopotentials. Phys. Rev. B 1996, 54, 1703-1710.

(27) Resta, R. Quantum-Mechanical Position Operator in Extended Systems. Phys. Rev. Lett. 1998, 80, 1800-1803.

(28) Saitta, A. M.; Saija, F.; Giaquinta, P. V. Ab Initio Molecular Dynamics Study of Dissociation of Water under an Electric Field. Phys. Rev. Lett. 2012, 108, 207801.

(29) Vicario, C.; Monoszlai, B.; Hauri, C. P. GV/m Single-Cycle Terahertz Fields from a Laser-Driven Large-Size Partitioned Organic Crystal. Phys. Rev. Lett. 2014, 112, 213901.

(30) Mishra, P. K.; Vendrell, O.; Santra, R. Ultrafast Energy Transfer to Liquid Water by Sub-Picosecond High-Intensity Terahertz Pulses: An Ab Initio Molecular Dynamics Study. Angew. Chem., Int. Ed. 2013, 52, 13685-13687.

(31) Bertie, J. E.; Ahmed, M. K.; Eysel, H. H. Infrared Intensities of Liquids. 5. Optical and Dielectric Constants, Integrated Intensities, and Dipole Moment Derivatives of Water and Water-D2 at $22^{\circ} \mathrm{C}$. J. Phys. Chem. 1989, 93, 2210-2218.

(32) Ashihara, S.; Huse, N.; Espagne, A.; Nibbering, E.; Elsaesser, T. Vibrational Couplings and Ultrafast Relaxation of the O-H Bending Mode in Liquid H2O. Chem. Phys. Lett. 2006, 424, 66-70.

(33) Walrafen, G. E.; Fisher, M. R; Hokmabadi, M. S.; Yang, W. Temperature Dependence of the Low- and High-Frequency Raman scattering from Liquid Water. J. Chem. Phys. 1986, 85, 6970-6982.

(34) Thomas, M.; Brehm, M.; Fligg, R.; Vöhringer, P.; Kirchner, B. Computing Vibrational Spectra from Ab Initio Molecular Dynamics. Phys. Chem. Chem. Phys. 2013, 15, 6608-6622.

(35) Praprotnik, M.; Janežič, D.; Mavri, J. Temperature Dependence of Water Vibrational Spectrum: A Molecular Dynamics Simulation Study. J. Phys. Chem. A 2004, 108, 11056-11062.

(36) Martí, J.; Padro, J. A.; Guàrdia, E. Molecular Dynamics Simulation of Liquid Water along the Coexistence Curve: Hydrogen Bonds and Vibrational Spectra. J. Chem. Phys. 1996, 105, 639-649.

(37) Padro, J. A.; Marti, J.; Guardia, E. Molecular Dynamics Simulation of Liquid Water at 523 K. J. Phys.: Condens. Matter 1994, 6, 2283.

(38) Walrafen, G. E.; Chu, Y. C.; Piermarini, G. J. Low-Frequency Raman Scattering from Water at High Pressures and High Temperatures. J. Phys. Chem. 1996, 100, 10363-10372.
(39) Mizoguchi, K.; Hori, Y.; Tominaga, Y. Study on Dynamical Structure in Water and Heavy Water by Low-Frequency Raman Spectroscopy. J. Chem. Phys. 1992, 97, 1961-1968.

(40) Hasted, J.; Husain, S.; Frescura, F.; Birch, J. Far-Infrared Absorption in Liquid Water. Chem. Phys. Lett. 1985, 118, 622-625.

(41) Walrafen, G. E. Raman Spectral Studies of the Effects of Temperature on Water and Electrolyte Solutions. J. Chem. Phys. 1966, $44,1546-1558$.

(42) Souaille, M.; Smith, J. C. Molecular Dynamics Analysis of Charge Fluctuations Associated with Far-Infrared Absorption in Water. Mol. Phys. 1996, 87, 1333-1347.

(43) Bellissent-Funel, M.-C.; Teixeira, J. Dynamics of Water Studied by Coherent and Incoherent Inelastic Neutron Scattering. J. Mol. Struct. 1991, 250, 213-230.

(44) Teixeira, J.; Bellissent-Funel, M. C.; Chen, S. H.; Dorner, B. Observation of New Short-Wavelength Collective Excitations in Heavy Water by Coherent Inelastic Neutron Scattering. Phys. Rev. Lett. 1985, $54,2681-2683$

(45) Krishnamurthy, S.; Bansil, R.; Wiafe-Akenten, J. Low-Frequency Raman Spectrum of Supercooled Water. J. Chem. Phys. 1983, 79, $5863-5870$

(46) Benedict, W. S.; Gailar, N.; Plyler, E. K. Rotation-Vibration Spectra of Deuterated Water Vapor. J. Chem. Phys. 1956, 24, 11391165.

(47) Larsen, N. Microwave Spectra of the Six Mono $-{ }^{13} \mathrm{C}$-Substituted Phenols and of some Monodeuterated Species of Phenol. Complete Substitution Structure and Absolute Dipole Moment. J. Mol. Struct. 1979, 51, 175-190.

(48) Herzberg, G. Electronic Spectra and Electronic Structure of Polyatomic Molecules; Van Nostrand: New York, 1966.

(49) Gertner, B. J.; Whitnell, R. M.; Wilson, K. R.; Hynes, J. T. Activation to the Transition-State - Reactant and Solvent Energy-Flow for a Model SN2 Reaction in Water. J. Am. Chem. Soc. 1991, 113, 7487.

(50) Benjamin, I.; Gertner, B. J.; Tang, N. J.; Wilson, K. R. Energy Flow in an Atom Exchange Chemical Reaction in Solution. J. Am. Chem. Soc. 1990, 112, 524-530. 